\title{
Smart-Aloha for Multi-hop Wireless Networks
}

\author{
Harkirat Singh and Suresh Singh \\ Department of Computer Science \\ Portland State University \\ Portland, OR 97207 \\ Email: \{harkirat, singh\}@cs.pdx.edu
}

\begin{abstract}
This paper presents a novel slotted ALOHAbased protocol for use in ad hoc networks where nodes are equipped with adaptive array smart antennas. The protocol relies on the ability of the antenna and DoA (Direction of Arrival) algorithms to identify the direction of transmitters and then beamform appropriately to maximize SINR (Signal to Interference and Noise Ratio) at the receiver. The performance of the protocol is evaluated using analytical modeling as well as detailed simulation in OPNET and Matlab where we demonstrate the benefits of using smart antennas. The impact of using different number of antenna elements is also studied for this environment.
\end{abstract}

\section{INTRODUCTION}

Smart antennas (or adaptive array antennas) have some unique properties that enable us to achieve high throughputs in ad hoc network scenarios. A transmitter equipped with a smart antenna can form a directed beam towards its receiver and a receiver can similarly form a directed beam towards the sender thus resulting in very high gain. A receiver can also identify the direction of multiple simultaneous transmitters by running DoA (Direction of Arrival) algorithms and use this information to determine the directions in which it should place nulls. Placing nulls effectively cancels out the impact of interfering transmitters. In this paper we enhance the standard slotted-Aloha protocol by adding beamforming and nulling capabilities provided by smart antennas. This new protocol is called Smart-Aloha. We use simulations as well as analysis to quantify the performance of SmartAloha and, as we will show, the throughputs achieved are very high and are better than some recent directional MAC protocols. Finally, we study the fairness properties of our protocol and show that, under a variety of singlehop and multi-hop scenarios, our protocol achieves fairness.

To motivate the use of smart antennas, it is useful to enumerate the additional capabilities provided by these

This work is funded by the NSF under grant ANIR-0125728. antennas over and above those provided by directional antennas alone.

1) Silencing Interferers: If a receiver knows that there are interfering transmitters in its neighborhood, it can form a directed beam towards the sender while simultaneously placing nulls in the direction of the other transmitters. A null effectively cancels the received signal power from a transmitter (even if the interferer is more powerful than the desired transmitter) and ensures a high SINR at the receiver.

2) Enhanced Neighbor Discovery: Identifying the direction of neighbors is necessary for transmission (so as to beamform appropriately) as well as for reception (to silence interferers). Earlier directional antenna papers typically use some form of sequential polling to identify the direction of one-hop neighbors [1]. Thus, for a $45^{\circ}$ sectored antenna, there are eight directions in which a node will periodically poll neighbors. Smart antennas can considerably ease the complexity of this task as follows.

An idle node receives all transmissions on its antenna and can run DoA algorithms to determine the direction of active transmitters. This is illustrated in Figure 1 where an idle node determines that there are two directions where there are active transmitters. It can then poll each of these directions actively to identify the node ids (note that nodes $a$ and $b$ both lie in close angular direction while $c$ lies in a different direction). This antennaassisted approach can reduce the message cost of the polling algorithm.

3) Flexible Beamforming: A smart antenna system can be configured as an omni-directional antenna or as a directional antenna with variable beamwidths (limited by the number of antenna elements, section II) and with arbitrarily precise 
boresight ${ }^{1}$. This flexibility allows us to explore the protocol space with arbitrary combinations of beamwidths for collision avoidance and data transmission.

The remainder of this paper is organized as follows. In the next section, we present a self-contained overview of adaptive antenna arrays. Section III summarizes the previous work in this and related areas. In section IV we describe Smart-Aloha in detail. Section V presents our simulation results and provides a comparison with the results of other authors. We also analyze the fairness of our two protocols in section V-B. Section VI presents our analytical model for the protocol which demonstrates the correctness of our simulation results. Finally, we summarize the main results in section VII.

\section{OVERVIEW of SMART Antennas}

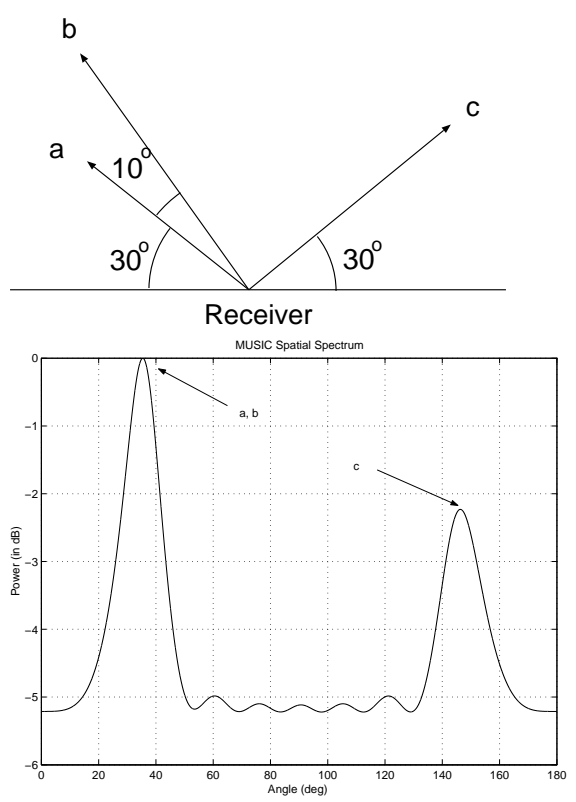

Fig. 1. Direction of Arrival (DoA).

We assume that each smart antenna (also called an adaptive antenna array) system is composed of a linear array of $M$ elements. Figure 2 provides a schematic of the smart antenna system. As illustrated in the figure, the antenna consists of $M$ antenna elements separated from each other by a known distance $d$. We can assume that a transmitter is located far enough away from the receiver that all the signals $S_{i}(t)$ arriving at the different antenna elements are parallel. However, since the elements are separated by distance $d$, the phase of the different signals

\footnotetext{
${ }^{1}$ Sectored antennas, for example, are relatively inflexible in this regard which can cause more collisions for nodes that lie outside the $3 \mathrm{~dB}$ beamwidth of the main beams.
}

is different. Let $w_{i}$ denote the phase and gain that is added to each signal $S_{i}(t)$. Then $z(t)$, the output sent to the receiver, can be written as,

$z(t)=A \sum_{i=1}^{M} w_{i} S_{i}(t)=A \sum_{i=1}^{M} w_{i} S_{0}(t) e^{-j \beta i d \cos \theta}+N(t)$ where, $N(t)$ is AWGN (Additive White Gaussian Noise), $\beta=2 \pi / \lambda$ is the phase propagation factor, $\lambda$ is the wavelength, and $A$ is an arbitrary gain constant. The weights $w_{i}$ used in this paper only shift the phase of the signal and leave the amplitude untouched. The representation for the weights is,

$$
w_{i}=e^{j \beta i d \sin \theta_{i}}
$$

For a more comprehensive discussion, please see [2]. In Figure 3 we show the different antenna patterns formed by a linear array of $M=8$ and 16 elements when the desired direction is $\theta=45^{\circ}$. We note that as the number of elements increases, the beamwidth becomes narrower. However, observe that rather than one beam, using a linear array results in two beams. We define the effective beamwidth as the sum of the beamwidth of the two beams formed by our antenna array.

A beneficial feature of these antennas is their ability to form nulls in given directions. For a single interferer, this is done by carefully shifting the phase $w_{i}$ of the received interfering signal at each antenna element $i$ and then adding these signals so that they cancel each other out. However, since we typically need to form several nulls in addition to a directed beam, the weight selection needs to be done carefully. In this study we select weights to maximize SINR at the receiver. One restriction to note is that, given $M$ elements, an antenna can form upto $M-1$ nulls. However, the shape of the desired beam can change depending on the number of and the direction of the nulls. Figure 4 illustrates two cases when using $M=8$ antenna elements with $\theta=45^{\circ}$ being the desired direction. In the first case, we are forming only two nulls $\left(25^{0}\right.$ and $\left.70^{\circ}\right)$ whereas in the second case we are forming six nulls $\left(10^{\circ}, 20^{\circ}, 30^{\circ}, 70^{\circ}, 80^{\circ}, 90^{\circ}\right)$. As can be seen, the shape of the beam and the direction of maximum gain changes dramatically.

Finally, smart antenna systems provide us with the ability to determine the direction of multiple transmitters. Many different DoA algorithms [2] have been developed over the past years and Figure 1 provides a typical output produced by a commonly used algorithm called MUSIC (MUltiple SIgnal Classification [2]). Here, there are three transmitters $a, b$ and $c$. The receiver can distinguish two directions from which it is receiving the transmissions. However, since $a$ and $b$ are very close in angular direction, it is hard to distinguish 


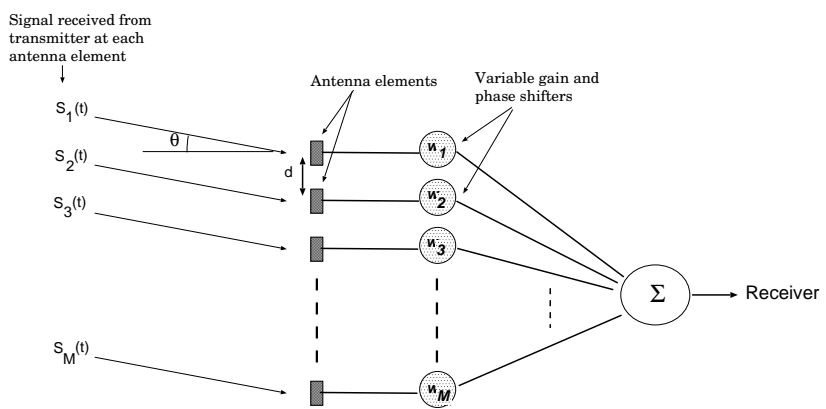

Fig. 2. Schematic of the smart antenna model used here.
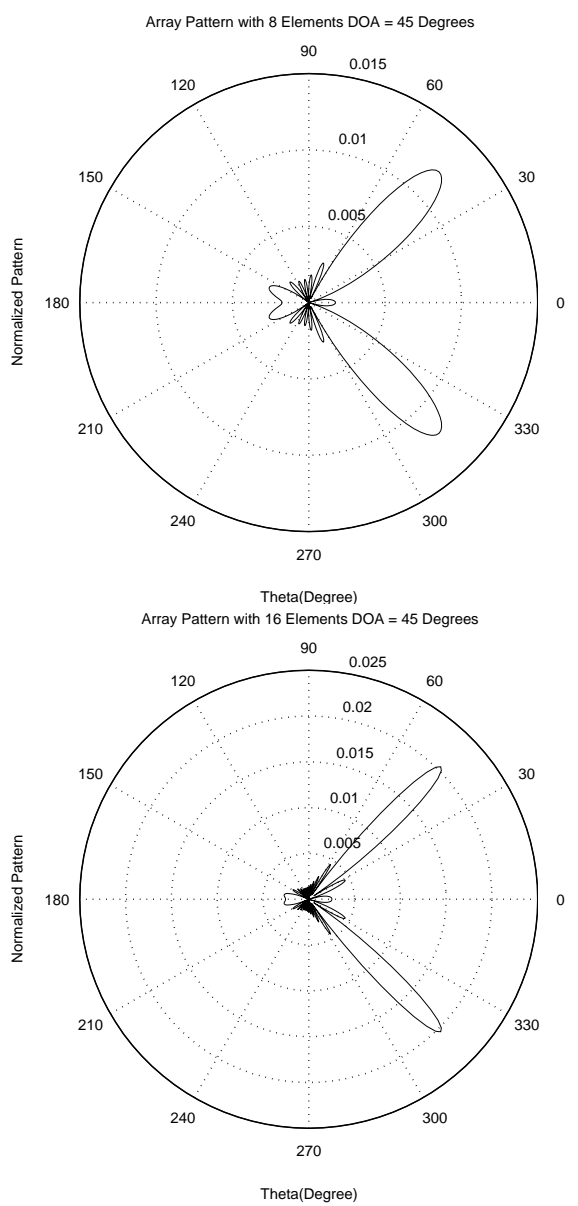

Fig. 3. Antenna patterns for 8 and 16 antenna elements.

between them. Using the DoA capability, a receiver can effectively place nulls in the directions of all the interfering transmitters and thus boost the SINR of its desired signal.

\section{LITERATURE REVIEW}

Recently there have been several papers that have looked at the problem of MAC design for ad hoc networks where nodes are equipped with directional antennas. The directional antenna models used include switched beam antennas (the antenna is sectored and one
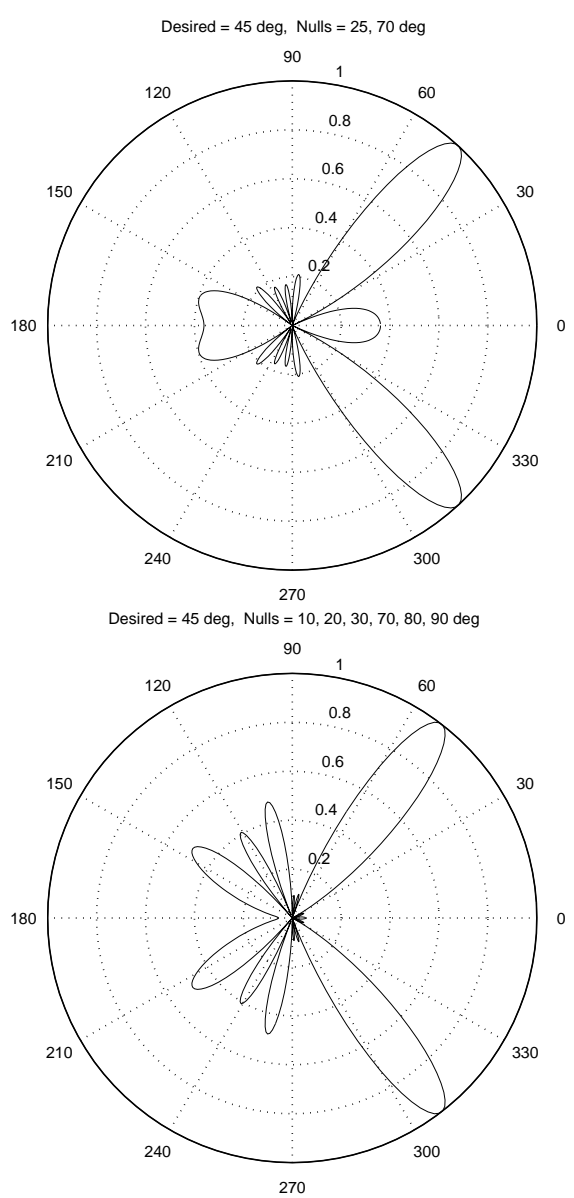

Fig. 4. Patters with 8 antenna elements and 2 or 6 nulls.

of these sectors is used depending on the direction of the communicating node), multi-beam antennas (here more than one beam can be used simultaneously), and adaptive antenna arrays (here the beam can be made to point in any direction as described in section II). Table I provides a summary of the main throughput results of the different protocols discussed below.

Among the papers that have studied the application of adaptive antenna arrays in ad hoc networking are [5], [8], [10]. [10] provides a high-level discussion of some promising research areas when using adaptive antenna arrays in ad hoc networks. They however do not provide any results. [5] examines the interaction and integration of several critical components of adaptive antenna arrays for use in ad hoc networks. This paper focuses on the design of these antennas for mobile devices with operating frequency of 20Ghz. The paper reports results of detailed OPNET simulations using a TDMA version of the 802.11 protocol in a single-hop network. A maximum throughput of 9 pkts/packet-time was achieved using the $8 \times 8$ array in a single-hop network with 55 nodes.

[8] studies the performance of Spatial TDMA and CSMA/CA in multihop ad hoc networks using adaptive 


\begin{tabular}{|c|c|c|c|c|c|c|c|}
\hline Prior Work & $\begin{array}{l}\text { Characteristics of } \\
\text { Simulation Expts. }\end{array}$ & \multicolumn{6}{|c|}{ Maximum Throughput } \\
\hline \multirow[t]{4}{*}{$\overline{[3]}$} & \multirow{4}{*}{$\begin{array}{l}\text { Switched beam antenna } \\
45^{0} \text { beamwidth, } 10 \mathrm{~dB} \text { gain, } 250 \mathrm{~m} \\
\text { range for omni, } 900 \mathrm{~m} \text { directional } \\
4 \mathrm{CBR} \text { sources, } 75 \mathrm{kbps}-2 \mathrm{Mbps} \text { each }\end{array}$} & \multicolumn{3}{|c|}{ Random Topology } & \multicolumn{3}{|c|}{ Mesh Topology } \\
\hline & & \multicolumn{6}{|c|}{$(\mathrm{N}=25,4$ hops $)$} \\
\hline & & MMAC & DMAC & 802.11 & MMAC & DMAC & 802.11 \\
\hline & & $\begin{array}{c}1000 \mathrm{kbps} \\
(5 \mathrm{x})\end{array}$ & $\begin{array}{l}400 \\
(2 \mathrm{x})\end{array}$ & $\begin{array}{l}200 \\
(1 \mathrm{x})\end{array}$ & $\begin{array}{l}800 \\
(4 \mathrm{x})\end{array}$ & $\begin{array}{c}300 \\
(1.5 \mathrm{x})\end{array}$ & $\begin{array}{l}200 \\
(1 \mathrm{x})\end{array}$ \\
\hline \multirow[t]{4}{*}{$\overline{[4]}$} & \multirow{4}{*}{$\begin{array}{l}\text { Multi-beam antenna } \\
(1,2,4 \text { beams each) } \\
30^{\circ} \text { beamwidth, } 2 \mathrm{Mbps} \text { channel } \\
\text { slotted ( } 8 \mathrm{~ms} \text { slot), } 16 \mathrm{Kbit} \text { packet } \\
\text { (Throughput converted to } \\
\text { bps from pkts/slot/net) }\end{array}$} & \multicolumn{3}{|c|}{$\begin{array}{l}\text { Fully connected } \\
\text { (20 nodes) }\end{array}$} & \multicolumn{3}{|c|}{$\begin{array}{c}\text { Multi-hop } \\
\text { (100 nodes, } 5 \text { hops) }\end{array}$} \\
\hline & & 1 beam & 2 & 4 & 1 & 2 & 4 \\
\hline & & 12Mbps & 30 & 60 & 60 & 150 & 300 \\
\hline & & & & $\mathrm{x}$ over $\mathrm{RO}$ & $\overline{A, U x D N}$ & & \\
\hline \multirow[t]{3}{*}{$\overline{\overline{[5]}}$} & \multirow{3}{*}{$\begin{array}{l}\text { Adaptive antenna; 4x4, 8x8 } \\
\text { planar arrays, TDMA-802.11, 1-hop }\end{array}$} & \multicolumn{3}{|c|}{$\overline{4 \times 4}$} & \multicolumn{3}{|c|}{$\overline{8 \times 8}$} \\
\hline & & \multicolumn{6}{|c|}{ (55 nodes) } \\
\hline & & \multicolumn{3}{|c|}{8 pkts/packet time } & \multicolumn{3}{|c|}{9 packets/packet time } \\
\hline \multirow[t]{3}{*}{ [6] } & \multirow{3}{*}{$\begin{array}{l}\text { Switched beam } \\
60^{\circ} \text { beamwidth }\end{array}$} & \multicolumn{2}{|c|}{ Proposed } & $\overline{\overline{\text { DRTS }}}$ & $\overline{\overline{C T S}}$ & \multicolumn{2}{|c|}{ CSMA/CA } \\
\hline & & \multicolumn{6}{|c|}{ (50 nodes) } \\
\hline & & \multicolumn{2}{|c|}{$3.5 \mathrm{Mbps}$} & \multicolumn{2}{|c|}{2.5} & \multicolumn{2}{|c|}{2} \\
\hline \multirow[t]{4}{*}{$\overline{\overline{[7]}}$} & \multirow{4}{*}{$\begin{array}{l}\text { Circular adaptive antenna array } \\
\text { beamwidth } 64^{0}, 8 \mathrm{~dB} \text { gain } \\
\text { (Improvement over } 802.11 \text { ) }\end{array}$} & \multicolumn{3}{|c|}{25 nodes (grid) } & \multicolumn{3}{|c|}{225 nodes (grid) } \\
\hline & & No PC & Global PC & Local PC & No PC & Global PC & Local PC \\
\hline & & & & $\overline{\mathrm{PC}}-\mathrm{Pow}$ & Control) & & \\
\hline & & $1.3 \mathrm{x}$ & $1.7 \mathrm{x}$ & $2.1 \mathrm{x}$ & $2.6 \mathrm{x}$ & $4.75 x$ & $5.25 x$ \\
\hline \multirow[t]{7}{*}{$\overline{[\overline{[8]}}$} & $\begin{array}{l}\text { Ideal adaptive antenna } \\
20 \text { nodes, no nulling }\end{array}$ & $\begin{array}{r}\operatorname{Pr} \\
\mathrm{O}-\mathrm{Omr}\end{array}$ & $\begin{array}{l}\text { col } \\
\text { irectional }\end{array}$ & & $\begin{array}{r}B e \\
20 \text { nodes }\end{array}$ & $\begin{array}{l}\text { width } \\
\text { egree }=7.5\end{array}$ & \\
\hline & & $\mathrm{D}-\mathrm{D}$ & ctional & $90^{\circ}$ & $60^{\circ}$ & $30^{\circ}$ & $10^{\circ}$ \\
\hline & (Improvement over omni case) & ORT & $\overline{\mathrm{DCTS}}$ & $35 \%$ & $57 \%$ & $100 \%$ & $142 \%$ \\
\hline & & $\overline{\text { DRT }}$ & $\overline{\mathrm{DCTS}}$ & $64 \%$ & $107 \%$ & $143 \%$ & $186 \%$ \\
\hline & Packet transmission is & DRT & DCTS & $28 \%$ & $43 \%$ & $\mathrm{n} / \mathrm{a}$ & $57 \%$ \\
\hline & directional at sender/receiver & ORT & DCTS & $29 \%$ & $50 \%$ & $86 \%$ & $121 \%$ \\
\hline & & & $\mathrm{MA}$ & $\mathrm{n} / \mathrm{a}$ & $400 \%$ & $\mathrm{n} / \mathrm{a}$ & $400 \%$ \\
\hline 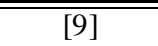 & 6-element circular antenna array & & & $\overline{\text { (No M }}$ & $\overline{\text { ility) }}$ & & \\
\hline & (10 fixed patterns - no adaptation) & Omni & $\mathrm{Rx} \operatorname{dir}$ & ional & DVCS & DVCs & Ideal \\
\hline & $45^{0}$ beamwidth, 100 nodes, $1500 \mathrm{~m}^{2}$ & & Tx Omni & ectional & & Rx Directio & \\
\hline & 2-ray propagation model, no nulling & $400 \mathrm{kbps}$ & & & $1.4 \mathrm{Mbps}$ & $2.2 \mathrm{I}$ & \\
\hline
\end{tabular}

TABLE I

Summary of DiRECTIONAL MAC PROTOCOL PERFormanCE.

antenna arrays. They examine the performance of different RTS/CTS schemes (DRTS/DCTS, ORTS/OCTS, DRTS/OCTS, etc.) on throughput. The main results indicate that narrower beamwidths $\left(10^{\circ}\right)$ do give the highest throughput though this value is not too different from the case when using $60^{\circ}$ beamwidths. In addition, they also performed simulations with dense as well as sparse networks. The highest throughput was achieved in dense networks (average degree 10.2) with lower throughput in sparse networks (average degree 6.9). This paper did not exploit the benefits of nulling and DoA as we do in our paper.

[4] develops slotted scheduling-based MAC protocols for nodes equipped with directional antennas. The directional antenna considered is a multi-beam adaptive array antenna (MBAA) which is capable of forming multiple beams. The protocols assume that nodes can engage in several simultaneous transmissions. Several recent papers have looked at MAC design using sectored directional antennas. [11] is one of the early papers which touches upon various ad hoc networking issues when using directional antennas. The authors discuss issues such as power control, hidden terminal problem, and which antenna models to use. [12] proposes a MAC protocol that uses directional antennas where mobile nodes do not have any location information. Each node is equipped with $M$ directional antenna elements. Each of the antenna elements has a conical pattern, spanning an angle of $2 \pi / M$ radians. The $M$ antennas at each node are fixed with non-overlapping beam directions so as to collectively span entire plane. The MAC protocol is assumed to be capable of switching any one or all the antennas to active or passive modes. In this work authors assume that all the antennas have same gain. The other assumption is that the transmitted signal will be completely attenuated outside the conical pattern of the directional antennas. 
The protocol uses omnidirectinal transmission of the RTS/CTS control packets. The receiver uses selection diversity, i.e. the receivers uses the signal from the antenna that is receiving maximum signal strength. The receiver also remembers the the antenna tha $t$ received the maximum power of the signal, thus, the receiver remembers the direction of the maximum power signal. The sender uses this information to directionally transmit the Data packet followed by directional exchange of the Ack. The directional transmission of unicast packet could reduce the interference at the overhearing no des, further, directional reception of the packets increases SINR (signal-to-interference-and-noise) at the receiver. The authors simulated a network of 225 nodes placed on a $15 \times 15$ uniform grid. Stationary as well as mobile node scenarios was examined. Using 4 antenna elements per node 2 to 3 times average throughput improvement over standard CSMA/CA with RTS/CTS was achieved. The paper did not examine the benefits of nulling or the impact of side-lobe interference. Furthermore, the propagation model was rather simplistic because of the assumption of complete attenuation outside the conical pattern. Omnidirectional transmission of the control pack ets reduces the potential of spatial reuse and increased channel capacity.

Similar to [12], [13], [14] consider a switched beam antenna mod el with has directional transmission range same as omnidirection. Further, they do not use nulling or narrower beamwidths. Motivated from the fact that directional antennas significantly increases the channel spatial reus e and results in higher channel capacity, they evolve a protocol which transmits CTS/Data/Ack directionally. Their protocol is somewhat motivated from PAMAS [15], which uses separate channel for control and data packet. [16] also develops MAC protocols for this scenario. They present a MAC protocol based on Directional RTS/CTS or a combination of DRTS/O-CTS (Omnidirectional CTS).

In [3] a multi-hop RTS is proposed to establish links between distant nodes. The direction in which the main lobe is to be oriented is determined by the MAC protocol (which in turn is provided this information by the network layer which is assumed to be neighbor-aware). The authors note that node alignment negates the benefits achieved due to directional antennas, however, unaligned routes enhances the spatial reuse. They show that their protocol has a 4-5x throughput as compared with 802.11. [7] describes the performance of 802.11 when using adaptive antenna arrays. Like [8], the authors consider the omni-RTS/omni-CTS followed by directional packet transmission within the context of 802.11. The transmit power for the data packet is smaller than that used for the RTS/CTS exchange and the authors present several power control variants. It is noteworthy that [3] also used 25-node grid networks but obtained a larger relative improvement (with respect to 802.11) in throughput compared with [7].

In [17], [18] the authors assume that each node maintains neighbor Angle-SINR table (AST) and they provide a link state based table-driven routing and MAC protocol. Based on AST a node calculates an affinity for an angle which provides maximum SINR. Based on this a NLS Table is formed. Nodes in the beamformed region remain in the omni mode but they make nulls in the direction of ongoing transmissions.

[19] investigates the performance of Smart Antennas in MIMO channels. [1] uses directional transmissions for control and data packets. It uses a directional-NAV table for transmission scheduling and collision avoidance. However, they do not exploit the capabilities of the smart antennas, such as beam steering and the placement of nulls in the direction of interferers.

In [9] 802.11 performance is studied with directional antennas. A circular antenna with 6 elements is assumed, and a node is capable of electronically steering the boresight towards a specific direction. A constant beamwidth of $45^{0}$ is assumed. However, it was observed that as the boresight changes, the side lobe pattern changes drastically. The key insight here is that the effects of side and back lobes cannot be ignored in the evaluation of network performance with directional antennas. [9] shows that using an ideal antenna results in a maximum throughput of $2.2 \mathrm{Mbps}$ while using a realistic antenna has a maximum throughput of only $1.4 \mathrm{Mbps}$. This fact, that antenna patterns matter in evaluating MAC behavior, is one that has largely been ignored by a great many authors who assume ideal antenna patterns.

Finally, we note that there have been several papers that look at the benefits of using smart antennas in cellular environments see, for instance, [20], [21], [22], [23], [24], [25], [26]. These papers look at models where the base station is equipped with one or multiple adaptive antenna arrays. Some authors [22] have examined the performance of Slotted-Aloha for these environments.

Our work here differs from all of the above papers in that, (1) all nodes use smart antennas for multi-hop networking, (2) our protocol exploits the DoA information obtained as well as the nulling capabilities of the antenna to enhance performance.

\section{DESCRIPTION OF SMART-Aloha}

Consider the case when a node $a$ needs to transmit a packet to node $b$ which is its one-hop neighbor. We 
assume that $a$ knows the angular direction of $b$ (as in [3]) and it can therefore form a beam in the direction of $b$. However, to maximize SINR, $b$ should also form a beam towards $a$ and form nulls in the direction of all other transmitters. In order to do this, $b$ needs to know two things - first, that $a$ is attempting to transmit to it, and second, the angular direction of all the other transmitters that interfere at $b$.

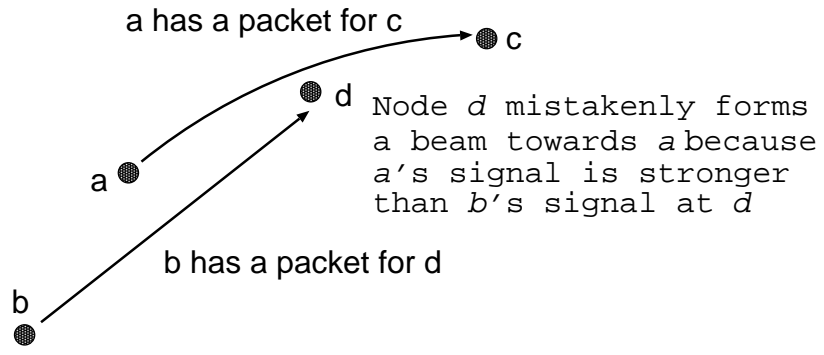

Fig. 5. False beamforming.

Smart-Aloha is a modified version of the standard Slotted-Aloha protocol. To transmit a packet, a transmitter forms a beam towards its receiver and begins transmission. However, it prefaces its packet transmission with the transmission of a short pure tone (this is a simple sinusoid). Idle nodes remain in an omnidirectional mode and receive a complex sum of all such tones (note that the tones are identical for all nodes and thus we cannot identify the nodes based on the tone) and run a DoA algorithm to identify the direction and strength of the various signals (Figure 1). An idle node then beamforms in the direction of the maximum received signal strength and forms nulls in other directions and receives the transmitted packet. If the receiver node was the intended destination for the packet, it immediately sends an ACK using the already formed directed beam. On the other hand, if the packet was intended for some other node, then the receiver discards it. A sender waits for an ACK immediately after transmission of the packet and if it does not receive the ACK, it enters backoff in the standard way. Thus, the Smart-Aloha protocol follows a Tone/Packet/Ack sequence.

The intuition behind the receiver beamforming in the direction of the maximum signal is that, because of the directivity of the antenna, there is a high probability that it is the intended recipient for the packet. However, we note that in cases, as in Figure 5, the receiver $d$ incorrectly beamforms towards $a$ because $a$ 's signal is stronger than $b$ 's. While this is not a serious problem in most cases, we can envision scenarios where the $b \longrightarrow d$ transmission gets starved due to a large volume of $a \longrightarrow$ $c$ traffic. An optimization we have therefore implemented is a single-entry cache scheme which works as follows:

\begin{tabular}{|l|l|}
\hline \multicolumn{2}{|c|}{ Simulation Parameters } \\
\hline Background Noise + ambient Noise & $-143 \mathrm{~dB}$ \\
Propagation model & Free space \\
Bandwidth & $1,000 \mathrm{kHz}$ \\
Min frequency & $2,402 \mathrm{MHz}$ \\
Data Rate & $2000 \mathrm{kbps}$ \\
Carrier Sensing Threshold & $+3 \mathrm{~dB}$ \\
Minimum SINR & $9 \mathrm{~dB}$ \\
Bit Error & Based on BPSK \\
& Modulation curve \\
Maximum radio range & $250 \mathrm{~m}$ \\
\hline \multicolumn{2}{|c|}{ Single Hop } \\
\hline Number of nodes Multihop & 40 \\
Area & $200 \times 200 \mathrm{~m}$ \\
\hline \multicolumn{2}{|c|}{} \\
\hline Number of nodes & 40 \\
Sparse case average node degree & 7.3 \\
Dense case average degree & 11.3 \\
Area & $300 \times 1500 \mathrm{~m}$ \\
\hline
\end{tabular}

TABLE II

OPNET SIMULATION PARAMETERS.

- If a node beamforms incorrectly in a given timeslot, it remembers that direction in a single-entry cache.

- In the next slot, if the maximum signal strength is again in the direction recorded in the singleentry cache, then the node ignores that direction and beamforms towards the second strongest signal.

- If the node receives a packet correctly (i.e., it was the intended recipient), it does not change the cache.

- If it receives a packet incorrectly, it updates the cache with this new direction.

- If there is no packet in a slot from the direction recorded in the cache, the cache is reset.

This simple mechanism ensures that in cases similar to Figure 5, connections are not starved. However, we can construct more complex scenarios where a singleentry cache will fail to prevent starvation. In these cases, more sophisticated multiple-entry caching schemes are required. However, in our simulations, we only use the single-entry caching scheme because the probability of more complex scenarios resulting in starvation are very rare.

\section{PERformance Study}

We evaluated the performance of our protocol via simulation as well as analysis. In this section we describe the simulation-based results (obtained in OPNET) and section VI presents our analytical model. We note that there is a very good correspondence between the simulation data and the analytical results indicating that our simulations are correct. 
OPNET provides an excellent physical layer model but has a drawback in that it has a very idealistic directional antenna model. To overcome this drawback we implemented the smart antenna model (for a linear array of antenna elements) in Matlab and interfaced it with the physical layer of OPNET. We invoke Matlab to compute $w_{i}$ 's (section II) based on actual received signal strength $s_{i}(t)$ at each antenna element as obtained from OPNET. We also modified OPNET's radio pipeline stage with the simulation parameters displayed in Table II. Finally, we assume that nodes do not move and that nodes know the angular direction of their neighbors (as in [3]). However, we need to point out that this is not a requirement of our protocol as the neighborhood can be determined using the algorithm outlined in Section 1. Nodes cache DoA data for transmissions they hear and then selectively poll active directions to identify the nodes that lie there.

We simulated a 40-node single-hop network and a multi-hop network. In all cases, packets arrived at nodes according to a poisson process and destinations were randomly uniformly chosen from among the neighbors. We measured the throughput (packets/slot) as well as the end-to-end delay. The packet length was set at 1024 bytes. We have left out the $95 \%$ confidence intervals in the interests of clarity. In all cases, the CI were very tight and did not overlap.

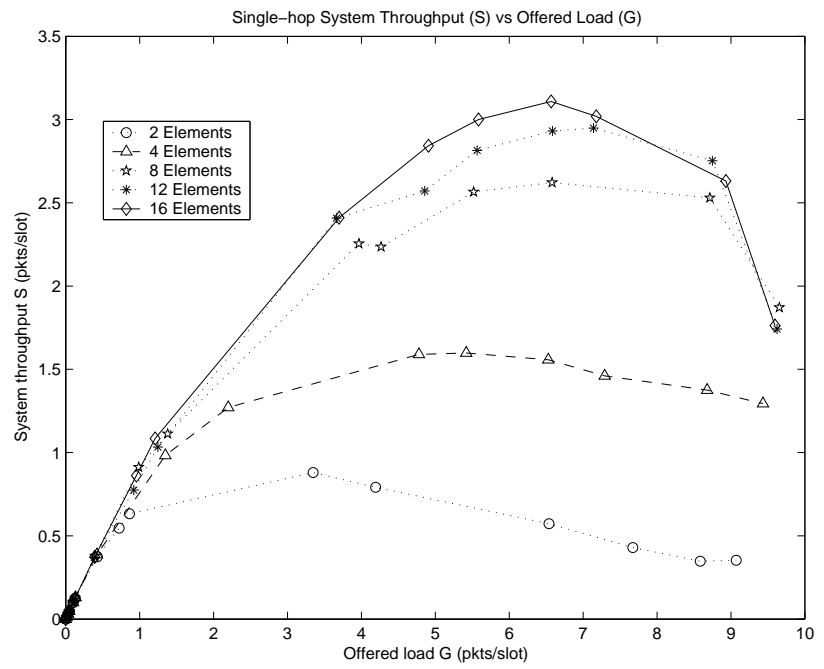

Fig. 6. Throughput for the single-hop case with 2, 4, 8, 16 antenna elements.

Figure 6 plots the average throughput as a function of load for the single-hop case and Figure 7 plots the average delay (slots). We vary load from a very low value of 0.001 packets/slot (network-wide) to 0.5 packets/slot. We vary the number of antenna elements from 2 to 16 . We observe that as the number of antenna

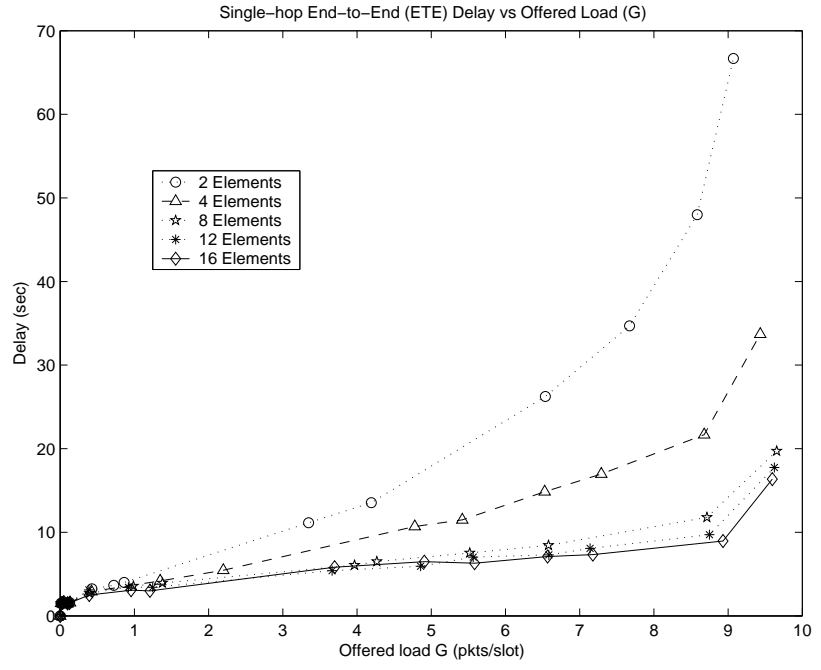

Fig. 7. ETE Delay for the single-hop case with 2, 4, 8, 16 antenna elements.

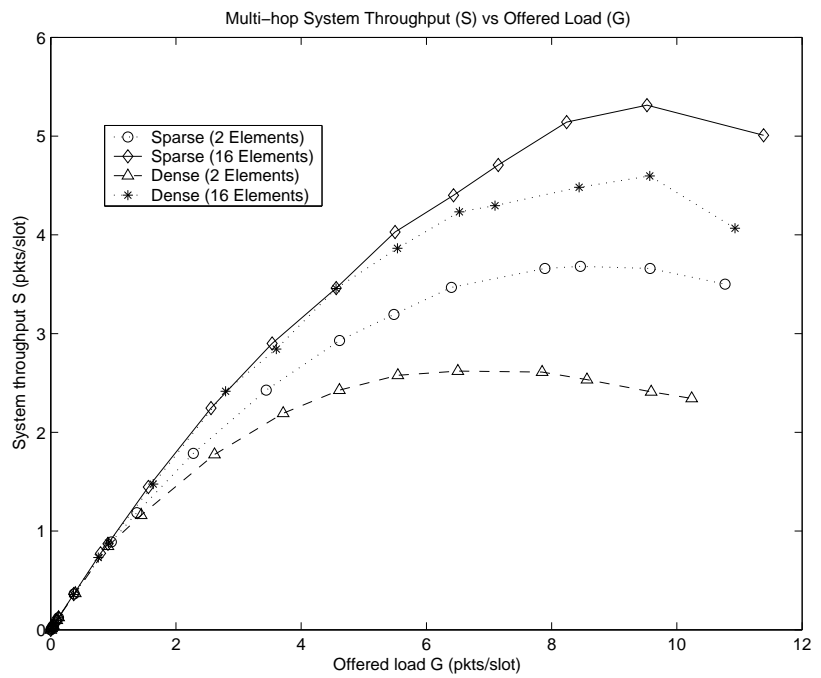

Fig. 8. Throughput for the multi-hop case with 2 and 16 antenna elements.

elements increases, the throughput increases from 1.7 to 3.1 packets/slot. This is because when we use larger number of antenna elements, the beamwidth becomes narrower, and we can form more nulls. Likewise, using larger number of antenna elements reduces the average delay because system capacity increases.

We examined the performance of the Smart-Aloha protocol for a multi-hop network as well. We considered two cases - dense network with an average degree of 11.3 and a sparse network with an average degree of 7.3. Figure 8 plots the throughput versus load for the dense network and for the sparse network cases when using 2 or 16 antenna elements. We observe that, in general, increasing the number of antenna elements increases throughout. For the sparse case, the improvement is 
approximately $1.8 \mathrm{x}$ while for the dense case, the improvement is almost $1.4 \mathrm{x}$. The relative increase is greater in the sparse case because nodes are more widely spaced and, there is a greater potential for the spatial reuse. However, after a point, there is no benefit to reducing the beamwidth. In the dense case, on the other hand, due to similar reasons the throughput is lower for both 2 and 16 antenna element cases.

\section{A. Performance Study in other Topologies}

In order to highlight the benefits of Smart-Aloha, we consider other single-hop and multi-hop topologies. The first set of experiments conducted uses a $5 \times 5$ mesh with pre-defined flows [3]. Figure 9 shows the network topology and flows used for two of these experiments. For the third experiment, we used a random node placement on the grid where a node's position is randomly shifted in the $\mathrm{x}$-axis and $\mathrm{y}$-axis by adding a displacement randomly selected from $[-150 \mathrm{~m},+150 \mathrm{~m}]$ and the flows are as in Figure 9(b). The traffic is CBR (Constant Bit Rate) which increases (per flow) from $75 \mathrm{kbps}$ to $2 \mathrm{Mbps}$. The packet size is 512 bytes. We used 10 different cases for random flows (Fig 9(b)) and randomly selected nodes. Some of the early results of this set of experiment were presented in [27],[28].

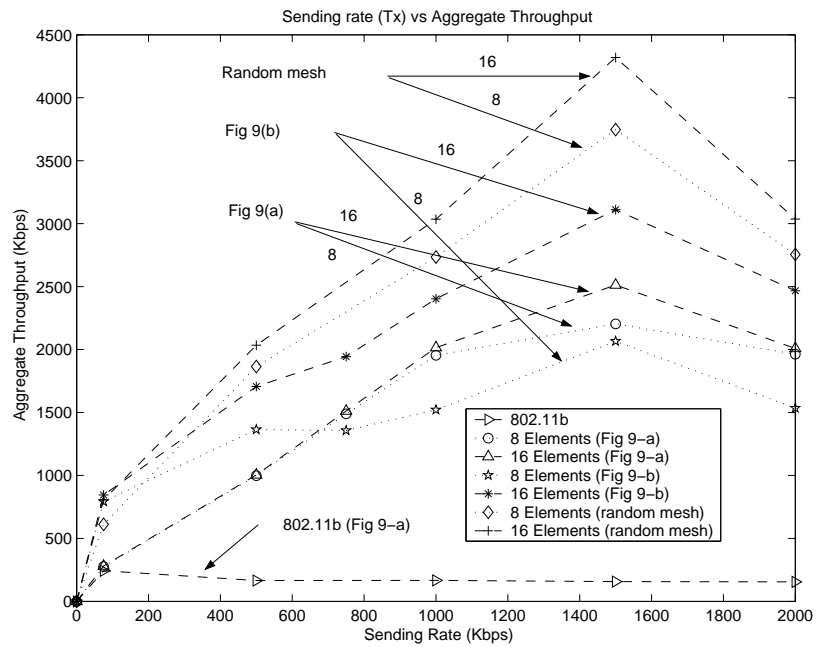

Fig. 10. Performance of Smart-Aloha when using the three topologies of [3].

Figure 10 plots the aggregate throughput of $802.11 \mathrm{~b}$ (with omni-directional antenna) and Smart-Aloha as a function of the data rate of one flow (for the two topologies in Figure 9 and for the random mesh case) using either 8 or 16 antenna elements. The performance of the 802.11 b protocol was alike in all the three topologies, hence, for the sake of the clarity of the graph we are plotting the results for Figure 9(a). We observe that using 16 antenna elements as opposed to 8 elements makes a big difference in aggregate throughput. This is because the beamwidth when using 16 elements is smaller than when using 8 elements which results in more simultaneous sessions/slot. For the flows in Figure 9(a), (when flows are aligned), we did not notice much difference in the performance of 16 and 8 antenna elements but for Figure 9(b) and for random topologies we do see a significant difference. The reason is that when flows are not aligned, there is a greater potential for spatial reuse with 16 antenna elements (due to its smaller beamwidth). Table III summarizes the results of this set of experiments.

The second set of the experiments were conducted in the single-hop case with 20 nodes and 5-hop cases with 100 nodes in a setting identical to [4] including use of $16 \mathrm{~KB}$ packets. We used 16 antenna elements for this study. Figure 11 plots the aggregate throughput as a function of arrival rate for the single-hop and multihop cases. We note that Smart-Aloha achieves a high of approximately $10.5 \mathrm{Mbps}$ as compared with the $1 \mathrm{Mbps}$ obtained by $802.11 \mathrm{~b}$ protocol. In fact, the throughput of Smart-Aloha increases with the arrival rate because of good spatial reuse of the channel. For the 100-node 5-hop case (Figure 11) 802.11b reaches a maximum throughput of well below $0.5 \mathrm{Mbps}$ while Smart-Aloha reaches maximum of $60 \mathrm{Mbps}$. Again, the better spatial reuse of the channel given the directivity of the antenna is the reason for this performance improvement.

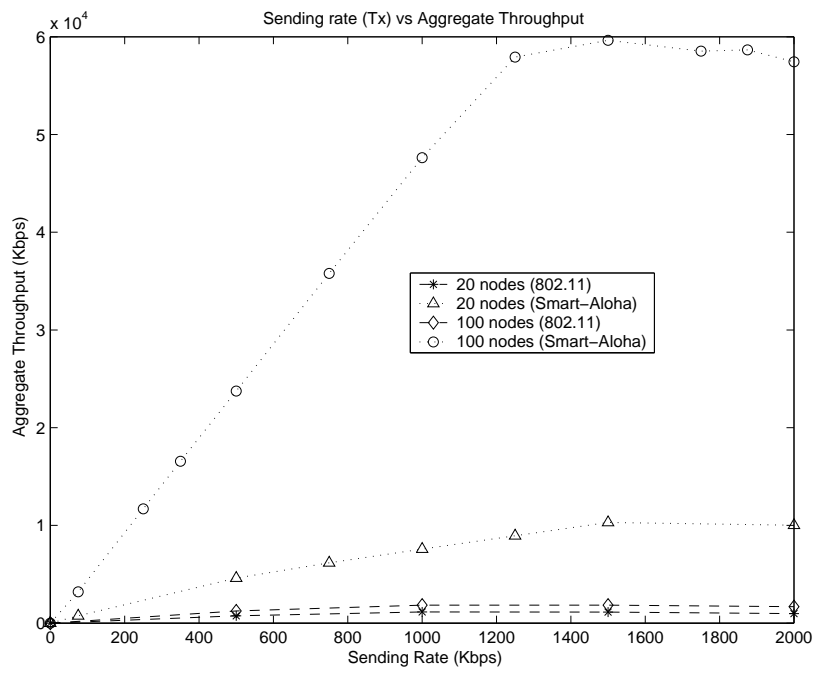

Fig. 11. Comparison with the single-hop 20-node case and 100-node multihop case in [4].

\section{B. Fairness of Smart-Aloha}

We performed a study of the fairness properties of Smart-Aloha and 802.11b using prior work [29], [30], 


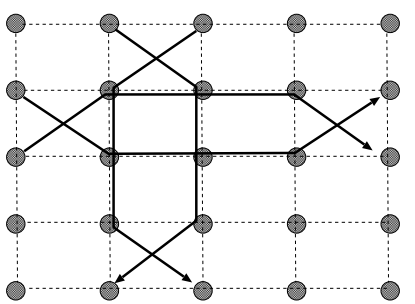

(a) Four flows (some alignment)

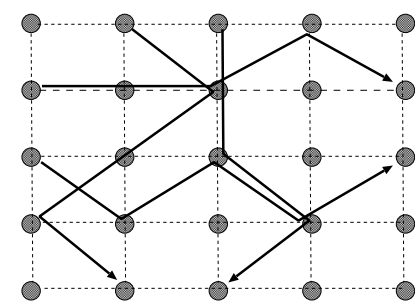

(b) Randomly selected flows

Fig. 9. 5x5 grid topology used to compare performance with [3].

\begin{tabular}{|l|l|l|l|l|l|l|}
\hline & \multicolumn{2}{|c|}{ Mesh Figure 9(a) } & \multicolumn{2}{c|}{ Mesh Figure 9(b) } & \multicolumn{2}{c|}{ Random Mesh } \\
\hline & $\begin{array}{l}16 \text { Elements } \\
\left(\sim 40^{\circ}\right)\end{array}$ & $\begin{array}{l}8 \text { Elements } \\
\left(\sim 70^{\circ}\right)\end{array}$ & $\begin{array}{l}16 \text { Elements } \\
\left(\sim 40^{\circ}\right)\end{array}$ & $\begin{array}{l}\text { 8 Elements } \\
\left(\sim 70^{\circ}\right)\end{array}$ & $\begin{array}{l}16 \text { Elements } \\
\left(\sim 40^{\circ}\right)\end{array}$ & $\begin{array}{l}8 \text { Elements } \\
\left(\sim 70^{\circ}\right)\end{array}$ \\
\hline Smart-Aloha & $2500 \mathrm{kbps}$ & 2150 & 3000 & 2000 & 4250 & 3750 \\
\hline
\end{tabular}

TABLE III

[31] as a guide. We considered the flows illustrated in Figure 12 where the dotted lines between two nodes in the figures indicates that the two nodes can hear one another. The arrows indicate the direction of flows and we used $2 \mathrm{Mbit} / \mathrm{sec}$ CBR traffic for each flow with 512 byte packets. The maximum channel capacity is also $2 \mathrm{Mbit} / \mathrm{sec}$ and the remaining parameters were set as per Table II.

Table IV shows the data rate achieved by each flow in each of the three topologies from Figure 12. In Topology 1 , nodes 1 and 2 are within range of one another and node 1 is in fact in the second symmetric lobe formed by node 2 towards node 3. In all cases, we note that SmartAloha results in fair channel sharing between the various flows even though the linear antenna array we use forms two main lobes that can cause unintentional interference. In addition to the topologies discussed above, we studied other topologies including the star topology with four transmitters sending to one common receiver (as in [31]). We note that all the flows shared the channel equally in this case as well.

\section{Throughrut \& Delay Analysis}

In order to validate our simulation, we developed an analytical model of Smart-Aloha as described in this section. The initial part of our derivation, where we set up the basic Markov chain, (equations 1 - 5) closely follows [22]. However, the key probabilities (equation 6 onwards) we develop are unique to Smart-Aloha. [22] uses a cellular network model where the base station has a smart antenna and can receive transmissions from at most one mobile in one slot. A later paper [32] generalized this to the case when the base station had multiple receivers. In our case, however, we consider an ad hoc network where a node can transmit to any

\begin{tabular}{|c|c|c|}
\hline \multicolumn{3}{|c|}{ Topology 1 } \\
\hline Flow & $\begin{array}{c}802.11 b \\
(\mathrm{Mbps})\end{array}$ & $\begin{array}{c}\text { Smart-Aloha } \\
(\mathrm{Mbps})\end{array}$ \\
\hline $0 \longrightarrow 1$ & 0.661 & 1.957 \\
$2 \longrightarrow 3$ & 0.663 & 1.978 \\
\hline \hline \multicolumn{3}{|c|}{ Topology 2 } \\
\hline Flow & $802.11 b$ & Smart-Aloha \\
\hline $0 \longrightarrow 1$ & 0.089 & 1.958 \\
$2 \longrightarrow 3$ & 0.108 & 1.827 \\
$4 \longrightarrow 5$ & 0.567 & 1.931 \\
\hline \hline \multicolumn{3}{|c|}{ Topology 3 } \\
\hline Flow & $802.11 b$ & Smart-Aloha \\
\hline $0 \longrightarrow 1$ & 0.427 & 1.573 \\
$2 \longrightarrow 3$ & 0.433 & 1.459 \\
$4 \longrightarrow 5$ & 0.430 & 1.896 \\
\hline
\end{tabular}

TABLE IV

AVERAGE DATA RATES OF DIFFERENT FLOWS.

of its neighbors and the probability of success depends on (1) whether the intended receiver is idle (i.e., not transmitting and not receiving from another node), (2) whether the receiver beamforms towards the sender during the slot (recall the problem of false beamforming Figure 5), and (3) the nulling capability of the receiver's antenna. Thus, we note that the derivation presented here is different from earlier works and is novel.

Consider a finite population of $M$ nodes each of which is equipped with a smart antenna. A node can be in one of two states - blocked and unblocked. In an unblocked state, the node transmits a packet in a slot with probability $p_{n}$ and in the blocked state the node transmits a packet with probability $p_{r}$. The destination of the packet is randomly uniformly chosen from the remaining $M-1$ nodes. Given this model, we can quantify system state in slot $k$ by a variable $0 \leq X_{k} \leq M$ which denotes the number of blocked nodes. We can thus describe 

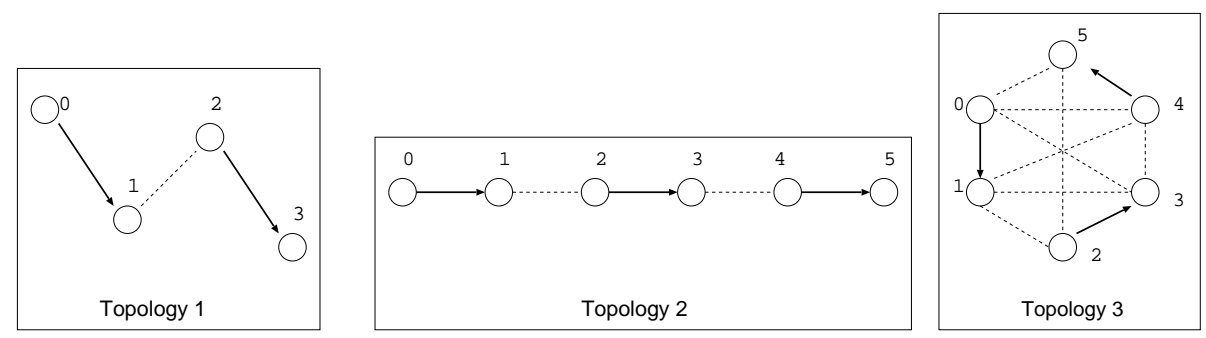

Fig. 12. Topologies used for fairness study (Topology $1 \& 2$ is multi-hop and Topology 3 is single-hop).

the time varying behavior of the system as a Markov chain where the state denotes the number of blocked terminals. Let $\mathcal{P}=\left[P_{i j}\right]$ denote the one-step transition matrix for this Markov chain and let $\pi=\left\{\pi_{i}\right\}$ denote the equilibrium probabilities of state $i$.

In a given slot the total number of packet transmissions can be written as $n_{t}=n_{r}+n_{n}$ where $n_{r}$ denotes the number of retransmissions and $n_{n}$ denotes the number of new transmissions. Following [22] for equations 1 5 , we can thus write,

$$
\begin{array}{r}
Q_{n}(l \mid i) \triangleq \operatorname{Pr}\left\{n_{n}=l \mid X_{k}=i\right\}=\left(\begin{array}{c}
M-i \\
l
\end{array}\right) \times \\
p_{n}^{l}\left(1-p_{n}\right)^{M-i-l}, i<M
\end{array}
$$

$Q_{r}(l \mid i) \triangleq \operatorname{Pr}\left\{n_{r}=l \mid X_{k}=i\right\}=\left(\begin{array}{c}i \\ l\end{array}\right) p_{r}^{l}\left(1-p_{r}\right)^{i-l}, i \geq$

Thus, the distribution of the total number of transmission in a slot can be written as,

$Q_{t}(l \mid i) \triangleq \operatorname{Pr}\left\{n_{t}=l \mid X_{k}=i\right\}=\sum_{s=0}^{l} Q_{n}(s \mid i) Q_{r}(l-s \mid i)$

Let us next determine the transition probabilities $P_{i j}$. We consider two cases: $j \leq i$ and $j \geq i$.

- $j=i-k, i=0, \ldots, M, k=0, \ldots, i$ : This can happen only if $n_{r} \geq k, n_{n} \geq 0$, and $n_{n}+k$ transmissions are successful. The probability of this can be written as,

$$
P_{i, i-k}=\sum_{s=0}^{M-i} \sum_{l=k}^{i} Q_{n}(s \mid i) Q_{r}(l \mid i) P_{s}(l+s, s+k)
$$

where, $P_{s}(a, b)$ is the probability of $b \leq a$ successful transmissions given $a$ total transmissions.

- $j=i+k, i=0, \ldots, M, k=0, \ldots, M-i$ : In order for this state transition to happen, exactly $k+l$ unblocked nodes need to become blocked and $l$ blocked nodes need to be successful. The

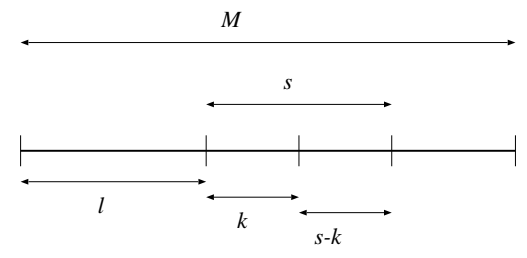

Fig. 13. Computing $P_{s}(l, k)$.

probability of this can be written as,

$$
P_{i, i+k}=\sum_{s=k}^{M-i} \sum_{l=0}^{i} Q_{n}(s \mid i) Q_{r}(l \mid i) P_{s}(l+s, s-k)
$$

To compute $P_{s}(l, k)$ we use the following simplified model: we assume that a node cannot receive a packet

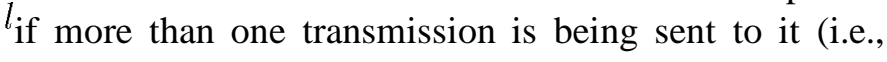
the packets identify this node as the receiver). However, even in this case the transmission may be unsuccessful if there are interfering transmissions that cannot be nulled by the receiver. In other words, a packet is successful if the node is able to make nulls in the direction of all the interfering transmitters (note that even if a node is not the receiver of a packet, it could still hear a transmission if it is in the directional beam formed by the transmitter). Thus, determining $P_{s}(l, k)$ reduces to (1) a combinatorial problem of determining the probability of there being $s \geq k$ receivers (that are not transmitting), (2) that all these $s$ nodes is the destination for one of the $l$ packets transmitted, and (3) the probability that $k$ out of $s$ receivers can correctly receive a packet given $(l-1)$ other transmissions, some of which may interfere (see Figure 13). Given that all nodes are unique, there are $(M-1)^{l}$ different ways in which $l$ packets can be transmitted. In order for there to be exactly $k$ successes, we must first identify $s$ nodes out of $M-l$ that will be the receivers. This can be done in $\left(\begin{array}{c}M-l \\ s\end{array}\right)$ ways. Of the $l$ transmissions, $s$ are transmitted to the $s$ selected receivers with the remaining $(l-s)$ transmitted to the $l$ transmitters themselves. Then, we can write, 


$$
P_{s}(l, k)=\left\{\begin{array}{l}
0, k>l, l+k>M,(l=1 \text { and } k=0) \\
1, l=k=1 \text { or } 0, \\
\frac{1}{(M-1)^{l}} \sum_{s=k}^{\min (l, M-l)}\left(\begin{array}{c}
M-l \\
s
\end{array}\right)\left(\begin{array}{l}
l \\
s
\end{array}\right) \\
\left(\begin{array}{c}
s \\
k
\end{array}\right) s !(l-1)^{l-s} p_{s}(l, E)^{k} \\
\left(1-p_{s}(l, E)\right)^{s-k}, l>1, l \geq k \geq 0
\end{array}\right.
$$

where, $p_{s}(l, E)$ is the probability of a successful packet reception given $l$ total transmissions and that the node can form $E$ nulls.

Consider Figure 14 where we show a transmission from node $a$ to node $b$. As shown, nodes $a$ and $b$ form beams towards each other of beamwidth $\theta$. Interference at node $b$ can occur in two different ways: (a) if there is a different transmitter within $b$ 's beam that is transmitting towards $b$ (i.e., node $c$ in Figure 14(b)), $b$ cannot form a null in that direction, (2) if there are at least $E+1$ transmitters outside $b$ 's beam that are transmitting towards $b$, there will be interference because $b$ can only form nulls towards $E$ transmitters. One other point to note is that, as shown in Figure 3, when a beam is formed in some direction, a second lobe is formed in a different direction. Thus, even if a node is not transmitting in $b$ 's direction, it can still cause interference due to the second lobe. Putting all these observations together, we now compute $p_{s}(l, E)$.

$p_{s}(l, E)=\sum_{\alpha=0}^{l-1}\left(\begin{array}{c}l-1 \\ \alpha\end{array}\right)\left(\frac{\theta}{2 \pi}\right)^{\alpha}\left(1-\frac{\theta}{2 \pi}\right)^{l-\alpha-1}$

$\times \operatorname{Pr}[$ No interference $-\alpha$ transmitters in receiver's beam $]$ $\times \operatorname{Pr}$ [No interference from $l-\alpha-1$ transmitters outside receiver's beam]

$$
\begin{aligned}
& =\sum_{\alpha=0}^{l-1}\left(\begin{array}{c}
l-1 \\
\alpha
\end{array}\right)\left(\frac{\theta}{2 \pi}\right)^{\alpha}\left(1-\frac{\theta}{2 \pi}\right)^{l-\alpha-1} \\
& \times\left(1-2 \frac{\theta}{2 \pi}\right)^{\alpha} \times \sum_{\beta=0}^{\min (E, l-\alpha-1)}\left(\begin{array}{c}
l-\alpha-1 \\
\beta
\end{array}\right)\left(\frac{\theta}{2 \pi}\right)^{\beta} \\
& \left(1-\frac{\theta}{2 \pi}\right)^{l-\alpha-\beta-1}
\end{aligned}
$$

In the above equation, we note that the probability that a transmitter's beam (or second lobe) is not pointed at a given receiver is $1-2 \frac{\theta}{2 \pi}$.

We can now use equations $4,5,6,7$ to write the transition matrix $\mathcal{P}$. Since the Markov chain is irreducible and all states are recurrent non-null and aperiodic, we can then solve the system of equations,

$$
\pi=\pi \mathcal{P} \text { and } \sum_{j=0}^{M} \pi_{j}=1
$$

to obtain the steady state probabilities $\pi_{j}$. Using these probabilities, it is trivial to determine the average system throughput $\bar{S}$ and average delay $\bar{D}$. For multihop networks with $N=M d$ nodes (d is the average degree), the average throughput can be written as $d \bar{S}$.

\section{A. Numerical Evaluation}

We compared our OPNET simulation (using values from Table II) against the analytical model developed above. Figure 15 plots the throughput versus load for a 10-node single-hop network with 8 antenna elements. As we can see, the simulation agrees very well with the analysis. For the multi-hop case, we considered a 40-node network with average degree comparable to the sparse case (Figure 8). Figure 16 plots the throughput versus load for this case when nodes have 16 element antennas. Again, the match between simulation and analysis is very good (at high loads it was impractical to obtain simulation data because of the large run times involved).

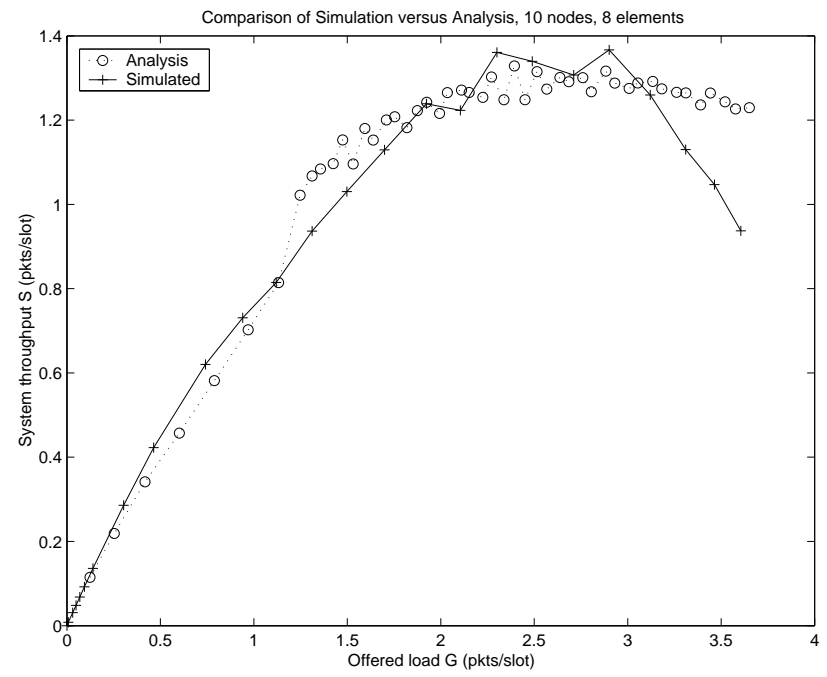

Fig. 15. Simulation vs Analysis for 10 nodes, single-hop.

\section{CONCLUSION}

This paper presents a simple tone-based protocol called Smart-Aloha for use with smart antenna systems. This protocol does not explicitly combat hidden terminals yet it shows very high throughput, exceeding that of many other protocols. We also demonstrate that our protocol shares the channel fairly among multiple competing flows. In the future, we will investigate the performance of Smart-Aloha in multipath environments and study the effect of training sequences on DoA algorithms for greater precision locationing. 

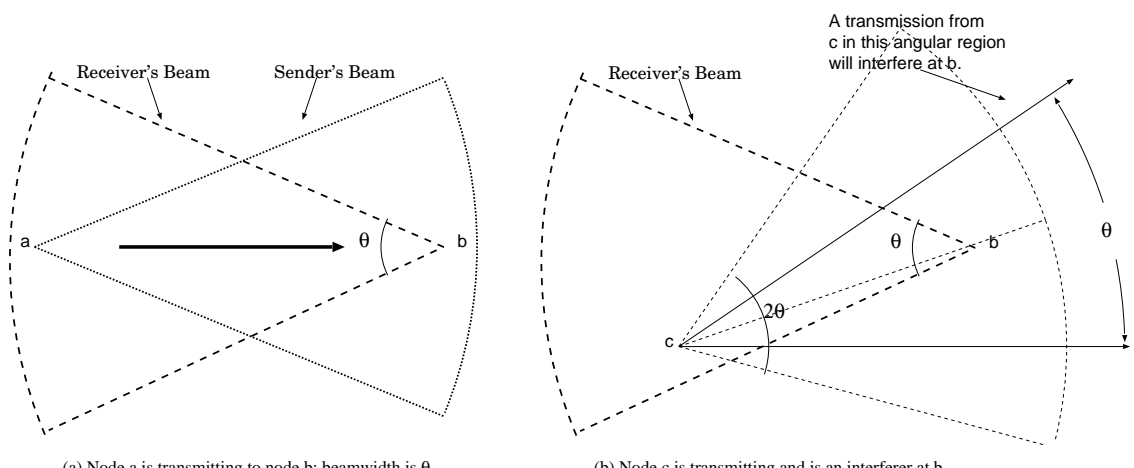

(b) Node $\mathrm{c}$ is transmitting and is an interferer at $\mathrm{b}$.

Fig. 14. Computing $p_{s}(l, E)$.

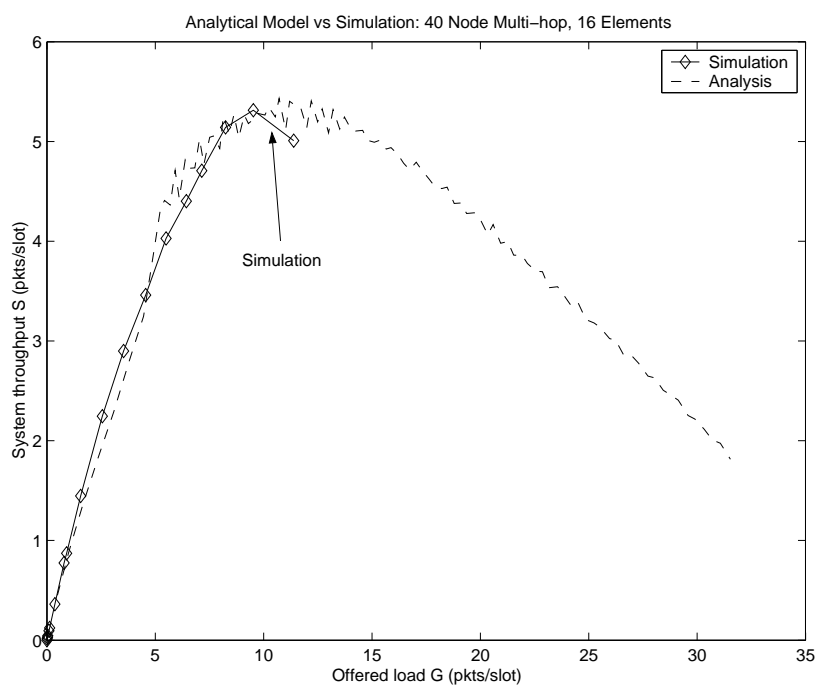

Fig. 16. Simulation vs Analysis for 40-nodes, multihop case.

\section{REFERENCES}

[1] Gentian Jakllari Thanasis Korakis and Leandros Tassiulas, "A mac protocol for full exploitation of directional antennas in adhoc wireless networks," in ACM Mobihoc'03, 1 - 3 June 2003.

[2] J. C. Liberti and T. S. Rappaport, Smart Antennas for Wireless Communications, Prentice Hall, 1999.

[3] Romit Roy Choudhury, Xue Yang, Ram Ramanathan, and Nitin H. Vaidya, "Using directional antennas for medium access control in ad hoc networks," in ACM/SIGMOBILE MobiCom 2002, 23 - 28 Sep 2002.

[4] Lichun Bao and J.J. Garcia-Luna-Aceves, "Transmission scheduling in ad hoc networks with directional antennas," in ACM/SIGMOBILE MobiCom 2002, 23 - 28 Sep 2002.

[5] S. Bellofiore, J. Foutz, R. Govindarajula, I. Bahceci, C. A. Balanis, A. S. Spanias, J.M. Capone, and T. M. Duman, "Smart antenna system analysis, integration, and performance for mobile ad-hoc networks (manets)," IEEE Transaction on Antennas and Propagation, vol. 50, no. 5, pp. 571 - 581, May 2002.

[6] T. ElBatt and B. Ryu, "On the channel reservation schemes for ad hoc networks utilizing directional antennas," in WPMC'02, 2002.

[7] N. Fahmy, T. D. Todd, and V. Kezys, "Ad hoc networks with smart antennas using 802.11-based protocols," in IEEE ICC'02.
[8] G. Marvin Sanchez, "Multiple access protocols with smart antennas n multihop ad hoc rural-area networks," M.S. thesis, Royal Institute of Technology, Sweeden, Radio Communication Systems Laboratory, Department of Signals, Sensors and Sytems, June 2002.

[9] Rajiv Bagrodia Mineo Takai, Jay Martin and Aifeng Ren, "Directional virtual carrier sensing for directional antennas in mobile ad hoc networks," in ACM/SIGMOBILE MobiHoc 2002, Oct 2002.

[10] Hend Koubaa, "Reflections on smart antennas for mac protocols in multihop ad hoc networks," in European Wireless'02, 25 28 Feb 2002.

[11] Ram Ramanathan, "On the performance of ad hoc networks with beamforming antennas," in ACM/SIGMOBILE MobiHoc 2001 , Oct 2001.

[12] J. You A. Nasipuri, S. Ye and R. Hiromoto, "A mac protocol for mobile ad hoc networks using directional antennas," in IEEE WCNC, 2000.

[13] Zhuochuan Huang and Chien-Chung Shen, "A comparison study of omnidirectional and directional mac protocols for ad hoc networks," in IEEE Globecom 2002, 2002.

[14] Chavalit Srisathapornphat Zhuochuan Huang, ChienChung Shen, "A mac protocol based on directional antenna and busy-tone for ad hoc networks," in IEEE MILCOM 2002, 2002.

[15] S. Singh and C. Raghavendra, "Pamas: Power aware multiaccess protocol with signalling for ad hoc networks," in $A C M$ Computer Communications Review, 1998.

[16] V. Shankarkumar Y.B. Ko and N.H. Vaidya, "Medium access control protocols using directional antennas in ad hoc networks," in IEEE INFOCOM 2000, March 2000.

[17] S. Horisawa S. Bandyopadhyay, K. Hausike and S. Tawara, "An adaptive mac and directional routing protocol for ad hoc wireless networks using espar antenna," in ACM/SIGMOBILE MobiHoc 2001, Oct 2001.

[18] S. Roy, D. Saha, S. Bandyopadhyay, T. Ueda, and S. Tanaka, "A network-aware mac and routing protocol for effective load balancing in ad hoc wireless networks with directional antenna," in ACM Mobihoc'03, 1 - 3 June 2003.

[19] Karthikeyan Sundaresan and Raghupathy Sivakumar, "On the medium access control problem in ad-hoc networks with smart antenna," in ACM Mobihoc'03, 1 - 3 June 2003.

[20] M. Zorzi and R.R. Rao, "Error control and energy consumption in communications for nomadic computing," in IEEE Transactions on Computers, March 1997.

[21] P.M. Soni A. Chockalingam, "Performance analysis of space diversity s-aloha with steerable beam smart antenna arrays," in IEEE GLOBECOM 'O0, Nov 2000. 
[22] James Ward and Jr. R. T. Compton, "Improving the performance of a slotted aloha packet radio network with an adaptive array," IEEE Transactions on Communications, vol. 40, no. 2, pp. 292 - 300, February 1992.

[23] A. S. Acampora, S. Krishnamurthy, and M. Zorzi, "Media access protocols for use with smart array antennas to enable wireless multimedia applications," in Proceedings 9th Tyrrhenian Workshop on Digital Communications, September 1997.

[24] C. Sakr and T. Todd, "Carrier-sense protocols for packetswitched smart antenna basestation," in ICNP'97, 1997.

[25] R.R. Rao A. Chockalingam, "Mac layer performance with steerable multibeam antenna arrays," in IEEE PIMRC '98, 1998.

[26] J. Zander, "Slotted aloha multihop packet radio networks with directional antennas," Electronics Letters, vol. 26, no. 25, pp. 2098-100, Dec. 1990.

[27] Harkirat Singh and Suresh Singh, "Doa-aloha: Slotted aloha for ad hoc networking using smart antennas," in IEEE VTC Fall'03, $6-9$ Oct 2003.

[28] Harkirat Singh and Suresh Singh, "A mac protocol based on adaptive beamforming for ad hoc networks,' in IEEE Pimrc'03, 7 - 10 Sep 2003.

[29] T. Nandagopal, T-E. Kim, X. Gao, and V. Bharghavan, "Achieving mac layer fairness in wireless packet networks," in $A C M$ MOBICOM'00, August 2000, pp. 87 - 98.

[30] B. Bensaou, Y. Wang, and C. C. Ko, "Fair medium access in 802.11 based wireless ad-hoc networks," in ACM MOBIHOC'00, August 2000, pp. $99-106$.

[31] V. Bharghavan, A. Demers, S. Shenker, and L. Zhang, "Macaw: A media access protocol for wireless lans," in ACM SIGCOMM'94, August 1994, pp. 212 - 225.

[32] James Ward and Jr. R. T. Compton, "High throughput slotted aloha packet radio networks with adaptive arrays," IEEE Transactions on Communications, vol. 41, no. 3, pp. 460 - 470, March 1993. 